\title{
Chemistry of New Zealand Apiaceae: A Rare Phenylpropanoid and Three New Germacrane Derivatives from Anisotome lyallii
}

\author{
Christian Zidorn ${ }^{\mathrm{a}, *}$ and Nigel B. Perry ${ }^{\mathrm{b}}$ \\ a Institut für Pharmazie, Abteilung Pharmakognosie, Universität Innsbruck, Innrain 52, \\ A-6020 Innsbruck, Austria. Fax: +43-512-507-2939 \\ E-mail: Christian.H.Zidorn@uibk.ac.at \\ b Plant Extracts Research Unit, New Zealand Institute for Crop \& Food Research Limited, \\ Department of Chemistry, University of Otago, Box 56, Dunedin, New Zealand \\ * Author for correspondence and reprint requests. \\ Z. Naturforsch. 56c, 951-956 (2001); received June 6/July 27, 2001
}

Apiaceae, Germacranes, Phenylpropanoids

A phytochemical investigation of the New Zealand endemic Apiaceae species Anisotome lyallii Hook.f. yielded (+)- $\alpha$-angeloyloxylatifolone (1), 6-O-angeloyl-8-O-tigloyl-6 $\beta, 8 \alpha, 11$-trihydroxygermacra-1(10) $E, 4 E$-diene (2), 6-O-tigloyl-8-O-tigloyl-6 $\beta, 8 \alpha, 11$-trihydroxygermacra$1(10) E, 4 E$-diene (3) and 6-O-tigloyl-8-O-tigloyl- $1 \alpha, 6 \beta, 8 \alpha, 11$-tetrahydroxygermacra- $4 E, 10$ (14)diene (4). The structures were elucidated by HR mass spectrometry and 1D- and 2DNMR spectroscopy. A chemosystematic survey for compounds 1-3 in other New Zealand Apiaceae by HPLC-MS revealed that 1-3 were confined to A. haastii Cockayne \& Laing and $A$. lyallii, and that some minor compounds in other species of Anisotome were isomers of 2 and $\mathbf{3}$.

\section{Introduction}

A. lyallii, a herb of up to $45 \mathrm{~cm}$ height, is an endemic species of coastal areas of the southern third of the New Zealand South Island and Steward Island (Dawson, 1961). A chemotaxonomic survey by HPLC-MS revealed that $A$. lyallii contains anisotomenes (Zidorn et al., submitted), irregular diterpenes, which have recently been discovered in sub-alpine members of the New Zealand and Tasmanian endemic genus Anisotome (van Klink et al., 1999).

HPLC-MS investigations also revealed the presence of three major unidentified compounds in $A$. lyallii. We now report the isolation and structure elucidation of these compounds and their distribution within the genus Anisotome.

\section{Results}

Air-dried whole plants of A. lyallii (298 g) were exhaustively extracted with acetone. The crude extract obtained after evaporating the solvent in vacuo $(36.2 \mathrm{~g})$ was further separated by repeated silica gel column chromatography (CC) using gradients of cyclohexane and acetone and subsequent Sephadex LH-20 CC with acetone as eluant. Enriched fractions of 1-4 (Fig. 1) were finally puri- fied by semi-preparative RP-18 HPLC using an isocratic mixture of acetonitrile and water containing $0.01 \%$ trifluoroacetic acid to yield $\mathbf{1}(10.0 \mathrm{mg})$, 2 (32.3 mg), 3 (63.1 mg) and 4 (4.0 mg).

Compound 1 was identified as $(+)$ - $\alpha$-angeloyloxylatifolone, a rare phenylpropanoid, on the basis of its on-line LC-mass spectrum $\{\mathrm{m} / z=307$ [M $+\mathrm{H}]^{+}(100), 339[\mathrm{M}+\mathrm{MeOH}+\mathrm{H}]^{+}(12), 371[\mathrm{M}$ $+2 \mathrm{MeOH}+\mathrm{H}]^{+}(18)$, congruent with a molecular formula of $\mathrm{C}_{16} \mathrm{H}_{18} \mathrm{O}_{6}$, its optical rotation and its ${ }^{1} \mathrm{H}$ NMR and ${ }^{13} \mathrm{C}$ NMR data. This compound so far has only been found in two other Apiaceae, Anthriscus sylvestris Hoffm. and Laserpitium siler L. (Stefanovic et al., 1977; Kozawa et al., 1978; Micovic et al., 1985). As ${ }^{13}$ C NMR data have not been published yet, these are given in the experimental section.

Compound 2 showed on-line mass signals at $\mathrm{m} /$ $z=419[\mathrm{M}+\mathrm{H}]^{+}$and $401\left[\mathrm{M}-\mathrm{H}_{2} \mathrm{O}+\mathrm{H}\right]^{+}$, appropriate for a molecular mass of $\mathrm{C}_{25} \mathrm{H}_{38} \mathrm{O}_{5}$. This molecular formula was verified by $\mathrm{HR}$ mass spectrometry. ${ }^{1} \mathrm{H}$ and ${ }^{13} \mathrm{C}$ NMR data (Tables I-II) revealed the presence of a sesquiterpene moiety and two hemiterpenic acid moieties. The sesquiterpene part of the molecule was identified on the basis of $1 \mathrm{D}$ - and $2 \mathrm{D}-\mathrm{NMR}$ experiments as a derivative of $6 \beta, 8 \alpha, 11$-trihydroxygermacra-1(10)E,4E- 

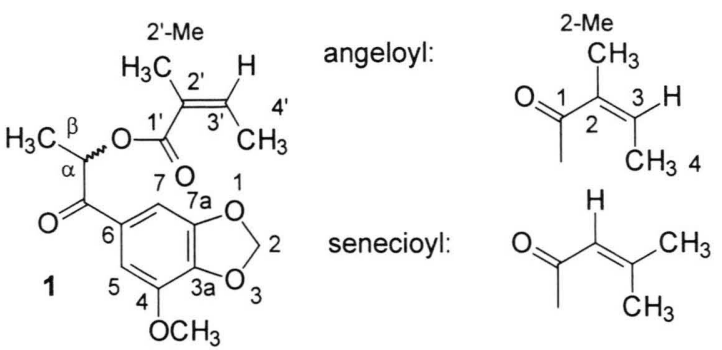

tigloyl:
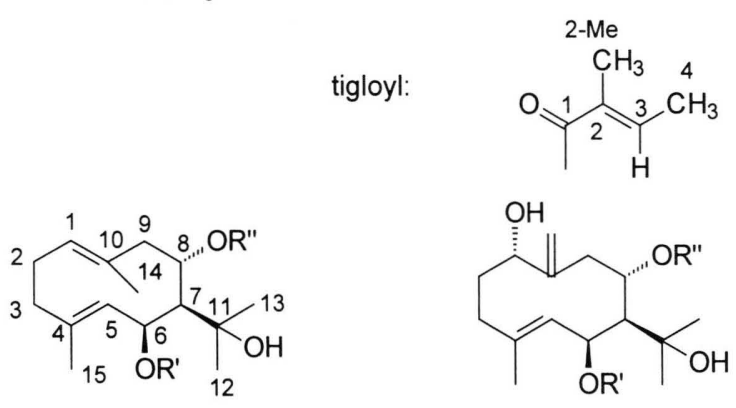

2: $R^{\prime}=$ angeloyl, $R^{\prime \prime}=$ tigloyl

3: $\mathrm{R}^{\prime}=\mathrm{R}^{\prime \prime}=$ tigloyl

4: $\mathrm{R}^{\prime}=\mathrm{R}^{\prime \prime}=$ tigloyl

5: $\mathrm{R}^{\prime}=\mathrm{H}, \mathrm{R}^{\prime \prime}=$ senecioyl

Fig. 1. Structures of a phenylpropanoid and four germacranes from Anisotome lyallii (1-4) and A. pilifera (5). (+)- $\alpha$-angeloyloxylatifolone (1), 6-O-angeloyl-8-O-tigloyl-6 $\beta, 8 \alpha, 11$-trihydroxygermacra-1(10) $E, 4 E$-diene (2), 6-O-tigloyl-8-O-tigloyl- $6 \beta, 8 \alpha, 11$-trihydroxygermacra-

$1(10) E, 4 E$-diene (3), 6-O-tigloyl-8-O-tigloyl- $1 \alpha, 6 \beta, 8 \alpha$,11 -tetrahydroxygermacra-4E,10(14)-diene (4), and 8 -O-senecioyl- $6 \beta, 8 \alpha, 11$-trihydroxygermacra-1(10)E, $4 E$ diene (5).

diene. NMR data of the sesquiterpene moiety were very similar to the data reported for 8 -Osenecioyl- $6 \beta, 8 \alpha, 11$-trihydroxygermacra-1(10) $E$,

$4 E$-diene 5 (Fig. 1 ), which we recently isolated from A. pilifera (Hook. f.) Cockayne \& Laing ( $\mathrm{Zi}$ dorn and Perry, submitted). The main difference between the spectra of $\mathbf{2}$ and $\mathbf{5}$ was the pronounced downfield-shift of the signal assignable to H-6 in $2\left(\delta_{\mathrm{H}}=5.80 \mathrm{ppm}\right.$ instead of $\left.4.91 \mathrm{ppm}\right)$ indicating esterification in that position. The hemiterpenic acid moieties in $\mathbf{2}$ were identified by ${ }^{1} \mathrm{H}$ NMR, ${ }^{13} \mathrm{C}$ NMR, HSQC and HMBC spectroscopy as angeloyl and tigloyl (Table II). The signal assignable to $\mathrm{H}-6$ showed a $\mathrm{HMBC}$ crosspeak to the carbonyl signal at $167.0 \mathrm{ppm}\left(\mathrm{C}-1^{\prime}\right)$ and the signal of $\mathrm{H}-8$ showed a crosspeak to the carbonyl signal at $166.5 \mathrm{ppm}\left(\mathrm{C}-1^{\prime \prime}\right)$. A signal indicative of an angeloyl moiety (H-3' at $6.04 \mathrm{ppm})$ showed a cross- peak to $\mathrm{C}-1^{\prime}$ at $167.0 \mathrm{ppm}$, and the signal of a proton indicative of a tigloyl moiety $\left(\mathrm{H}-3^{\prime \prime}\right.$ at 6.81 ppm) showed a crosspeak to C-1" at $166.5 \mathrm{ppm}$. Therefore, 2 was identified as 6-O-angeloyl-8-Otigloyl-6 $\beta, 8 \alpha, 11$-trihydroxygermacra-1(10) $E, 4 E$ diene, a previously unreported compound.

On-line and HR mass spectra of $\mathbf{3}$ were identical to those obtained for compound $\mathbf{2}$, suggesting that $\mathbf{3}$ was an isomer of $\mathbf{2}$. This was verified by ${ }^{1} \mathrm{H}$ and ${ }^{13} \mathrm{C}$ NMR spectroscopy (Table II), showing almost perfectly superimposable signals for the sesquiterpene moiety of the molecule, plus signals assignable to two tigloyl moieties. The signals of protons H-6 and H-8 showed HMBC crosspeaks to the carbonyls of the tigloyl moieties. Thus, compound 3 is 6-O-tigloyl-8-O-tigloyl-6 $\beta, 8 \alpha, 11$-trihydroxygermacra-1(10)E,4E-diene, another new compound.

HRMS data of $\mathbf{4}$ indicated a molecular formula of $\mathrm{C}_{25} \mathrm{H}_{38} \mathrm{O}_{6} \cdot{ }^{1} \mathrm{H}$ and ${ }^{13} \mathrm{C}$ NMR spectra (Tables I, II) showed signals assignable to a sesquiterpene moiety and two tigloyl moieties. Results from HSQC and HMBC experiments identified the sesquiterpene part of the molecule as a 1,6,8,11-tetraoxygermacra-4,10(14)-diene derivative. The ${ }^{1} \mathrm{H}$ NMR shifts of H-6 and H-8, and HMBC crosspeaks from the signals of these protons to the two carbonyl signals, revealed that the tigloyl moieties were attached to C- 6 and C-8. Therefore C- 1 and C-11 both bore hydroxyl groups.

NOE spectra showed two sets of interactions: between $\mathrm{H}-1, \mathrm{H}-5$ and $\mathrm{H}-8$; and between $\mathrm{H}-6, \mathrm{H}-$ 7 , one $\mathrm{H}-14$ and Me-15. This suggested that these two sets of protons were on opposite faces of the molecule. Assuming the usual absolute stereochemistry at C-7, this led to a proposed structure 4 with $1 \alpha$-OH, $6 \beta$-tigloyl and $8 \alpha$-tigloyl groupings. Conformational searching and molecular modeling of this proposed structure led to two predicted ring conformations (Fig. 2 and Table III) with various rotations around $\mathrm{C}-11-\mathrm{OH}$ and $\mathrm{C}-7-\mathrm{C}-11$. The major conformation (about $75 \%$ populated) explained the NOE interactions given above. However, this major conformation did not account for the observed NOE interaction between $\mathrm{H}-5$ and one or other $\mathrm{H}-9$, since these protons are over $0.44 \mathrm{~nm}$ apart in this conformation. In the minor conformation (about $25 \%$ populated), $\mathrm{H}-5$ and $\mathrm{H}$ $9 \alpha$ are $0.21 \mathrm{~nm}$ apart (Fig. 2), which would lead to a strong NOE interaction. Other evidence for the 
Table I. ${ }^{1} \mathrm{H}$ NMR data of compounds $2-\mathbf{4}^{\mathrm{a}}$.

\begin{tabular}{|c|c|c|c|}
\hline Position & 2 & 3 & 4 \\
\hline \multicolumn{4}{|c|}{ Sesquiterpene moiety } \\
\hline 1 & $5.161 \mathrm{H}, \mathrm{dq}(12.0,1.5)$ & $5.151 \mathrm{H}$, br d $(12.0)$ & $4.251 \mathrm{H}$, br d $(8.5)$ \\
\hline \multirow[t]{2}{*}{2} & $2.411 \mathrm{H}, \mathrm{m}$ & $2.391 \mathrm{H}, \mathrm{m}$ & $2.112 \mathrm{H}, \mathrm{m}$ \\
\hline & $2.151 \mathrm{H}, \mathrm{m}$ & $2.131 \mathrm{H}, \mathrm{m}$ & \\
\hline 3 & $2.182 \mathrm{H}, \mathrm{m}$ & $2.152 \mathrm{H}, \mathrm{m}$ & $2.142 \mathrm{H}, \mathrm{m}$ \\
\hline 5 & $5.131 \mathrm{H}, \mathrm{d}(7.0)$ & $5.131 \mathrm{H}$, br d $(6.5)$ & $5.241 \mathrm{H}$, br d (5.5) \\
\hline 6 & $5.801 \mathrm{H}$, br d $(6.0)$ & $5.771 \mathrm{H}$, br d $(6.0)$ & $5.821 \mathrm{H}$, br d $(5.5)$ \\
\hline 7 & $1.871 \mathrm{H}, \mathrm{m}$ & $1.861 \mathrm{H}, \mathrm{m}$ & $2.221 \mathrm{H}, \mathrm{dd}(4.5,2.5)$ \\
\hline 8 & $5.651 \mathrm{H}, \mathrm{dd}(12.0,6.0)$ & $5.691 \mathrm{H}, \mathrm{dd}(12.0,6.0)$ & $5.881 \mathrm{H}$, dd $(9.0,4.5,4.5)$ \\
\hline \multirow[t]{2}{*}{9} & $2.761 \mathrm{H}, \mathrm{dd}(13.0,6.0)$ & $2.731 \mathrm{H}, \mathrm{dd}(13.0,6.0)$ & $2.672 \mathrm{H}, \mathrm{m}$ \\
\hline & $1.981 \mathrm{H}, \mathrm{m}$ & $1.981 \mathrm{H}, \mathrm{dd}(13.0,12.0)$ & \\
\hline 12 & $1.443 \mathrm{H}, \mathrm{s}$ & $1.413 \mathrm{H}, \mathrm{s}$ & $1.373 \mathrm{H}, \mathrm{s}$ \\
\hline 13 & $1.393 \mathrm{H}, \mathrm{s}$ & $1.373 \mathrm{H}, \mathrm{s}$ & $1.293 \mathrm{H}, \mathrm{s}$ \\
\hline \multirow[t]{2}{*}{14} & $1.693 \mathrm{H}, \mathrm{b}$ & $1.693 \mathrm{H}, \mathrm{s}$ & $5.361 \mathrm{H}$, br s \\
\hline & & & $5.311 \mathrm{H}$, br s \\
\hline 15 & $1.553 \mathrm{H}, \mathrm{s}$ & $1.533 \mathrm{H}, \mathrm{s}$ & $1.553 \mathrm{H}, \mathrm{s}$ \\
\hline \multicolumn{4}{|c|}{ Hemiterpenic acid moiety I } \\
\hline $3^{\prime}$ & $6.041 \mathrm{H}$, qq $(7.5,1.5)$ & $6.661 \mathrm{H}, \mathrm{qq}(7.0,1.5)$ & $6.761 \mathrm{H}, \mathrm{qq}(7.5,1.0)$ \\
\hline $4^{\prime}$ & $1.953 \mathrm{H}, \mathrm{dq}(7.5,1.5)$ & $1.693 \mathrm{H}, \mathrm{m}^{\mathrm{b}}$ & $1.753 \mathrm{H}, \mathrm{q}(1.5)$ \\
\hline $2^{\prime}-\mathrm{Me}$ & $1.693 \mathrm{H}, \mathrm{b}$ & $1.673 \mathrm{H}, \mathrm{dq}(7.0,1.5)$ & $1.743 \mathrm{H}, \mathrm{dq}(7.5,1.0)$ \\
\hline \multicolumn{4}{|c|}{ Hemiterpenic acid moiety II } \\
\hline $3^{\prime \prime}$ & $6.81, \mathrm{qq}(7.0,1.0)$ & $6.851 \mathrm{H}, \mathrm{qq}(7.0,1.5)$ & $6.871 \mathrm{H}, \mathrm{qq}(7.5,1.0)$ \\
\hline $4^{\prime \prime}$ & $1.863 \mathrm{H}$, br s & $1.873 \mathrm{H}, \mathrm{q}(1.5)$ & $1.883 \mathrm{H}, \mathrm{q}(1.5)$ \\
\hline $2^{\prime \prime}-\mathrm{Me}$ & $1.813 \mathrm{H}, \mathrm{dq}(7.0,1.0)$ & $1.833 \mathrm{H}, \mathrm{dq}(7.0,1.5)$ & $1.833 \mathrm{H}, \mathrm{dq}(7.5,1.0)$ \\
\hline
\end{tabular}

a Measured in $\mathrm{CDCl}_{3}$ at $500 \mathrm{MHz}$, referenced to solvent residual signals of $\mathrm{CHCl}_{3}$ at $7.25 \mathrm{ppm}$.

b Overlapping signals.

Table II. ${ }^{13} \mathrm{C}$ NMR data of compounds $2-\mathbf{4}^{\mathrm{a}}$.

\begin{tabular}{|c|c|c|c|c|c|c|c|}
\hline Position & 2 & 3 & 4 & Position & 2 & 3 & 4 \\
\hline \multicolumn{4}{|c|}{ Sesquiterpene moiety } & \multicolumn{4}{|c|}{ Hemiterpenic acid moiety I } \\
\hline 1 & $132.1 \mathrm{~d}$ & $132.1 \mathrm{~d}$ & $87.0 \mathrm{~d}$ & $1^{\prime}$ & $167.0 \mathrm{~s}$ & $167.3 \mathrm{~s}$ & $167.0 \mathrm{~s}$ \\
\hline 2 & $24.8 \mathrm{t}$ & $24.8 \mathrm{t}$ & $28.6 \mathrm{t}$ & $2^{\prime}$ & $127.4 \mathrm{~s}$ & $128.7 \mathrm{~s}$ & $128.6 \mathrm{~s}$ \\
\hline 3 & $38.7 \mathrm{t}$ & $38.7 \mathrm{t}$ & $37.6 \mathrm{t}$ & $3^{\prime}$ & $139.6 \mathrm{~d}$ & $137.3 \mathrm{~d}$ & $138.0 \mathrm{~d}$ \\
\hline 4 & $135.7 \mathrm{~s}$ & $135.6 \mathrm{~s}$ & $134.9 \mathrm{~s}$ & $4^{\prime}$ & $15.7 \mathrm{q}$ & $11.7 \mathrm{q}$ & $11.9 \mathrm{q}$ \\
\hline 5 & $130.6 \mathrm{~d}$ & $130.5 \mathrm{~d}$ & $128.5 \mathrm{~d}$ & $2^{\prime}-\mathrm{Me}$ & $20.2 \mathrm{q}$ & $14.3 \mathrm{q}$ & $14.6 \mathrm{q}$ \\
\hline 6 & $70.7 \mathrm{~d}$ & $71.1 \mathrm{~d}$ & $71.2 \mathrm{~d}$ & & & & \\
\hline 7 & $52.9 \mathrm{~d}$ & $53.0 \mathrm{~d}$ & $50.0 \mathrm{~d}$ & \multirow{2}{*}{\multicolumn{4}{|c|}{ Hemiterpenic acid moiety II }} \\
\hline 8 & $75.2 \mathrm{~d}$ & $75.2 \mathrm{~d}$ & $71.0 \mathrm{~d}$ & & & & \\
\hline 9 & $40.9 \mathrm{t}$ & $40.9 \mathrm{t}$ & $43.1 \mathrm{t}$ & $1^{\prime \prime}$ & $166.5 \mathrm{~s}$ & $166.6 \mathrm{~s}$ & $166.5 \mathrm{~s}$ \\
\hline 10 & $129.6 \mathrm{~s}$ & $129.7 \mathrm{~s}$ & $142.9 \mathrm{~s}$ & $2^{\prime \prime}$ & $128.7 \mathrm{~s}$ & $128.6 \mathrm{~s}$ & $128.6 \mathrm{~s}$ \\
\hline 11 & $73.5 \mathrm{~s}$ & $73.5 \mathrm{~s}$ & $73.2 \mathrm{~s}$ & $3^{\prime \prime}$ & $137.5 \mathrm{~d}$ & $137.6 \mathrm{~d}$ & $138.3 \mathrm{~d}$ \\
\hline 12 & $30.4 \mathrm{q}$ & $30.2 \mathrm{q}$ & $29.7 \mathrm{q}$ & $4^{\prime \prime}$ & $12.3 \mathrm{q}$ & $12.3 \mathrm{q}$ & $12.3 \mathrm{q}$ \\
\hline 13 & $29.5 \mathrm{q}$ & $29.4 \mathrm{q}$ & $29.0 \mathrm{q}$ & $2^{\prime \prime}-\mathrm{Me}$ & $14.5 \mathrm{q}$ & $14.5 \mathrm{q}$ & $14.6 \mathrm{q}$ \\
\hline 14 & $20.7 \mathrm{q}$ & $20.7 \mathrm{q}$ & $118.6 \mathrm{t}$ & & & & \\
\hline 15 & $16.5 \mathrm{q}$ & $16.8 \mathrm{q}$ & $17.4 \mathrm{q}$ & & & & \\
\hline
\end{tabular}

${ }^{a}$ Measured in $\mathrm{CDCl}_{3}$ at $125 \mathrm{MHz}$, referenced to solvent signals of $\mathrm{CDCl}_{3}$ at $77.00 \mathrm{ppm}$. Multiplicities were derived from DEPT experiments.

presence of both ring conformations of $\mathbf{4}$ in $\mathrm{CDCl}_{3}$ solution was given by the couplings of the $\mathrm{H}-8$ signal. The major ring conformation (Fig. 2) has H-8 transoid to $\mathrm{H}-9 \alpha$, with a predicted coupling con- stant of $11.5 \mathrm{~Hz}$ (Table III). The actual largest $\mathrm{H}-8$ to $\mathrm{H}-9$ coupling constant for 4 was $9.0 \mathrm{~Hz}$ (Table II). This is in good agreement with the weighted mean of the $\mathrm{H}-8$ to $\mathrm{H}-9 \alpha$ coupling con- 

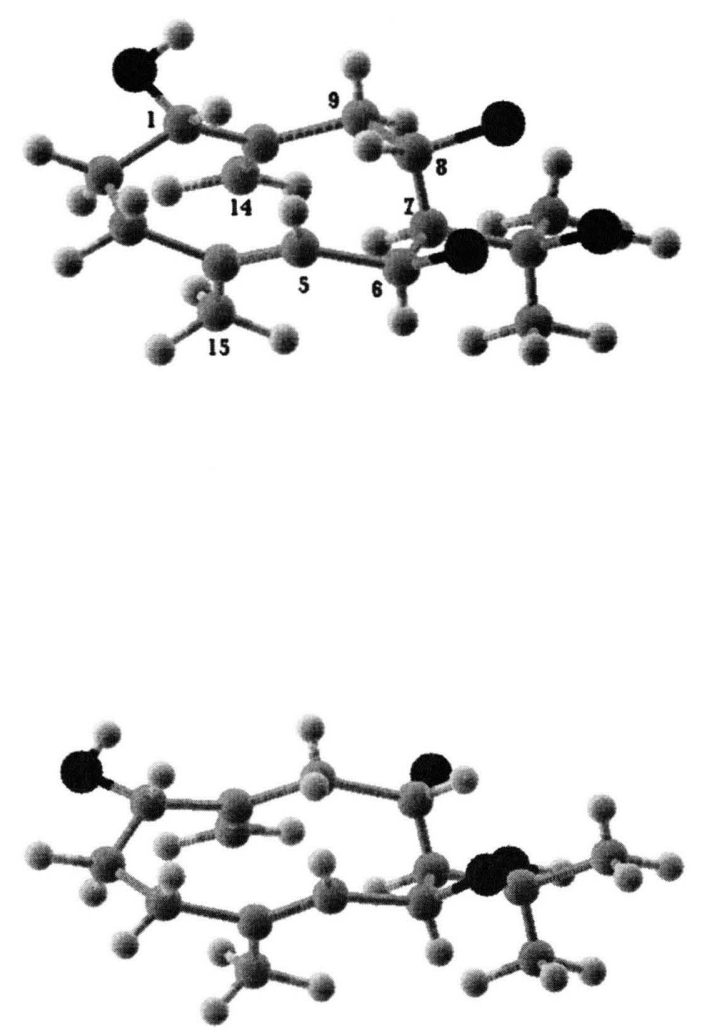

Fig. 2. Major (above) and minor (below) ring conformations predicted for germacrane $\mathbf{4}$ (tigloyl moieties omitted).

stants predicted for the main conformations ( $>1 \%$ populated) found by conformational searching and molecular modeling (Table III). These ring conformations seem to be exchanging rapidly at room temperature, since there was no sign of broadening in the ${ }^{13} \mathrm{C}$ NMR spectrum.

The $\alpha$-position of the hydroxy group at $\mathrm{C}-1$ was corroborated by a comparison of the ${ }^{1} \mathrm{H}$ NMR coupling pattern of $\mathrm{H}-1$ with literature data for $1 \alpha, 6 \beta$-dihydroxygermacra- $4 E, 10$ (14)-diene and $1 \beta, 6 \beta$-dihydroxygermacra-4E,10(14)-diene (Barrero et al., 1999). The minor ring conformation of 4 (Fig. 2) is similar to the predominant ring conformation of $1 \alpha, 6 \beta$-dihydroxygermacra- $4 E, 10(14)$ diene predicted by molecular modeling (Barrero et al., 1999). Consequently 4 is the new compound 6-O-tigloyl-8-O-tigloyl-1 $\alpha, 6 \beta, 8 \alpha, 11$-tetrahydroxygermacra-4E,10(14)-diene.

A survey for 1-3 (4 was hardly detectable in crude extracts of $A$. lyallii) by HPLC-MS in all seventeen known species of Anisotome (Dawson, 1961; Webb, 1986; Parsons et al., 1998) revealed that these compounds were confined to samples of $A$. haastii and A. lyallii. These analyses also revealed that compounds exhibiting the same online mass spectra as $\mathbf{2}$ and $\mathbf{3}$ but with slightly differing retention times occurred in A. aromatica Hook. f., in both varieties of $A$. imbricata (Hook.f.) Cockayne, and in $A$. lanuginosa (Kirk) J. W. Dawson. These isomers, which may be germacra-1(10)E,4Ediene-6,8,11-triol derivatives with different combinations of two hemiterpenic acids, were only present as minor compounds and were not isolated. We recently reported 8 -O-senecioyl- $6 \beta, 8 \alpha, 11$-trihydroxygermacra-1(10) E,4E-diene 5 from $A$. pilifera (Zidorn and Perry, submitted).

A vast number of different germacra$1(10) E, 4 E$-diene derivatives has been isolated from various plants. Interestingly, germacra$1(10) E, 4 E$-diene-6,8,11-triol derivatives have so far only been found in members of the genus Anisotome (this work; and Zidorn and Perry, submitted). Therefore germacranes with that particular substitution pattern are chemosystematic markers

Table III. Molecular modeling and selected NMR data for compound $\mathbf{4}$.

\begin{tabular}{|c|c|c|c|c|c|}
\hline \multirow{2}{*}{$\begin{array}{l}\text { Ring } \\
\text { Conformation }\end{array}$} & \multirow{2}{*}{$\begin{array}{l}\text { Energy } \\
(\mathrm{kcal} / \mathrm{mol})\end{array}$} & \multirow{2}{*}{$\begin{array}{l}\text { Population } \\
(\% \text { at } 300 \mathrm{~K})\end{array}$} & \multicolumn{3}{|c|}{ Proton-proton couplings $(\mathrm{Hz})$} \\
\hline & & & $\mathrm{H}-8-\mathrm{H}-7$ & $\mathrm{H}-8-\mathrm{H}-9 \alpha$ & H-8 - H-9ß \\
\hline Major & 42.858 & 36.40 & 1.1 & 11.5 & 3.4 \\
\hline Major & 43.045 & 26.60 & 1.1 & 11.5 & 3.4 \\
\hline Minor & 43.452 & 13.44 & 11.5 & 3.3 & 3.6 \\
\hline Minor & 43.550 & 11.41 & 11.5 & 3.3 & 3.6 \\
\hline Major & 44.056 & 4.88 & 1.1 & 11.5 & 3.4 \\
\hline Major & 44.649 & 1.81 & 1.1 & 11.5 & 3.4 \\
\hline Major & 44.907 & 1.17 & 1.1 & 11.5 & 3.4 \\
\hline Other & 44.927 & 1.13 & 11.5 & 3.2 & 3.7 \\
\hline Weighted mean & - & - & 3.8 & 9.0 & 3.3 \\
\hline Actual (in $\mathrm{CDCl}_{3}$ ) & - & - & 4.5 & 9.0 & 4.5 \\
\hline
\end{tabular}


for this umbelliferous genus, but are not detectable in all Anisotome species. A similar, but not correlated, pattern of patchy occurrence only in Anisotome has been found for the irregular anisotomene diterpenes (Zidorn et al., submitted). These observations reinforce taxonomic questions about the boundaries of this genus (Dawson, 1961).

\section{Experimental}

Plant material. - A. lyallii was collected at Cannibal Bay/Otago/New Zealand (S 46 $28^{\prime}$, E $169^{\circ} 46^{\prime}$, alt.: $10 \mathrm{~m}$ a.m.s.l.) in January 2000. A voucher specimen was deposited in the herbarium of the Plant Extracts Research Unit (voucher code: CZ-000118-3). Collection data of other Anisotome species investigated by HPLC-MS and extraction procedures and HPLC-MS parameters are described in full in Zidorn et al. (submitted). Air dried subaerial parts of the plants were ground, $500 \mathrm{mg}$ plant material was mixed with $10.0 \mathrm{ml}$ of a methanolic stock solution containing $0.200 \mathrm{mg} / \mathrm{ml}$ of $N$-phenyl-undecanamide (Perry et al., 1996) as internal standard and extracted three times with $25 \mathrm{ml}$ of methanol for $7.5 \mathrm{~min}$. with an IKA-25 Ultraturrax apparatus at 24000 cycles $/ \mathrm{min}$. Extracts were combined and brought to dryness in vacuo. The residue was dissolved in $4 \mathrm{ml}$ of methanol, filtered and used for HPLC analysis; HPLC system 1 (for quantification), oven temperature: $40{ }^{\circ} \mathrm{C}$; column: Zorbax Rx-C18, $4.6 \mathrm{~mm} \times 150 \mathrm{~mm}$, particle size $3.5 \mu \mathrm{m}$; guard column: Phenomenex $\mathrm{C} 18,4 \mathrm{~mm} \times 3.00 \mathrm{~mm}$; detection wavelength: $205 \mathrm{~nm}$; injection volume: $10 \mu \mathrm{l}$; mobile phase A: $0.01 \%$ trifluoroacetic acid in water; mobile phase B: $\mathrm{CH}_{3} \mathrm{CN}$; flow rate: $1.00 \mathrm{ml} / \mathrm{min}$; linear gradient: 0 min $80 \%$ A, $20 \%$ B; 5 min $80 \%$ A, $20 \%$ B; $10 \min 50 \%$ A, $50 \%$ B; $31 \min 46.5 \%$ A, $53.5 \%$ B; $55 \mathrm{~min} 5 \% \mathrm{~A}, 95 \% \mathrm{~B}$; stop time: $60 \mathrm{~min}$; post time: $15 \mathrm{~min}$; HPLC system 2 (for HPLC-MS analyses) oven temperature: $40^{\circ} \mathrm{C}$; column: Zorbax Rx-C18, $4.6 \mathrm{~mm} \times 150 \mathrm{~mm}$, particle size $3.5 \mu \mathrm{m}$; guard column: LiChroCart $4 \times 4 \mathrm{~mm}$ packed with $\mathrm{LiCh}-$ rospher RP-18 material ( $5 \mu \mathrm{m}$ particle size); detection wave length: $205 \mathrm{~nm}$; injection volume: $10 \mu \mathrm{l}$; mobile phase A: $0.15 \%$ acetic acid in water; mobile phase $\mathrm{B}$ : $0.15 \%$ acetic acid in $\mathrm{MeOH}$; flow rate: $1.00 \mathrm{ml} / \mathrm{min}$; linear gradient: $0 \mathrm{~min} 70 \% \mathrm{~A}$, $30 \% \mathrm{~B} ; 5 \min 70 \%$ A, $30 \% \mathrm{~B} ; 10 \min 40 \%$ A, $60 \%$
B; $31 \min 37.5 \%$ A, $63.5 \%$ B; $55 \min 2 \%$ A, $98 \%$ $\mathrm{B}$; stop time: $60 \mathrm{~min}$; post time: $15 \mathrm{~min}$. LC-MS analysis were performed with a Finnigan MAT SSQ 7000 mass spectrometer by APCI in the positive mode, employing a CID value of $-5 \mathrm{~V}$, a corona amperage of $5 \mu \mathrm{A}$, a sheath gas pressure of 50 PSI and a capillary temperature of $150{ }^{\circ} \mathrm{C}$.

The following retention times in HPLC-systems 1 and 2 were observed, respectively: $1(23.9,19.6)$, 2 (42.9, 39.8), 3 (39.9, 35.4), 4 (23.0, n.o.). The HPLC analyses for quantification were run in triplicate. The following amounts of compounds 1-4 were estimated in subaerial parts of $A$. lyallii by comparing the peak area of the internal standard and the peak areas of compounds 1-4: 1 (1.09 \pm $0.01 \mathrm{mg} / \mathrm{g}), 2(2.59 \pm 0.07), 3(1.17 \pm 0.02 \mathrm{mg} / \mathrm{g}), 4$ $(0.04 \pm 0.00 \mathrm{mg} / \mathrm{g})$.

Semi-preparative HPLC - Column: Phenomenex Luna $250 \times 10 \mathrm{~mm}(5 \mu \mathrm{m}$ particles, productnr.: 006-4252-N0), guard column: Phenomenex $\mathrm{C} 18,4 \mathrm{~mm} \times 3.00 \mathrm{~mm}$, flowrate: $5.00 \mathrm{ml}$, detection wavelength: $205 \mathrm{~nm}$, isocratic $50 \% \mathrm{MeCN}, 50 \%$ $\mathrm{H}_{2} \mathrm{O}+0.01 \%$ TFA (1, retention time: $20 \mathrm{~min} ; \mathbf{4}$, retention time: $17 \mathrm{~min}$ ); isocratic $68 \% \mathrm{MeCN}, 32 \%$ $\mathrm{H}_{2} \mathrm{O}+0.01 \%$ TFA (2, retention time: $20 \mathrm{~min} ; \mathbf{3}$, retention time: $18 \mathrm{~min})$.

NMR data of 1 (measured in $\mathrm{CDCl}_{3}$ at 500 and $125 \mathrm{MHz}$, respectively). ${ }^{1} \mathrm{H}$ NMR: phenylpropane moiety, $\delta_{\mathrm{H}}=7.27(\mathrm{~d}, \mathrm{~J}=1.5 \mathrm{~Hz}, 1 \mathrm{H}, \mathrm{H}-7), 7.14(\mathrm{~d}$, $\mathrm{J}=1.5 \mathrm{~Hz}, 1 \mathrm{H}, \mathrm{H}-5), 6.06$ (s, 2H, H-2), 5.91 (q, J = $7.5 \mathrm{~Hz}, 1 \mathrm{H}, \mathrm{H}-\alpha), 3.92(\mathrm{~s}, 3 \mathrm{H}, \mathrm{OMe}), 1.55(\mathrm{~d}, \mathrm{~J}=$ $7.5 \mathrm{~Hz}, 3 \mathrm{H}, \mathrm{H}-\beta)$; angeloyl moiety, $\delta_{\mathrm{H}}=6.12(\mathrm{dd}$, $\left.\mathrm{J}=7.5,1.5 \mathrm{~Hz}, 1 \mathrm{H}, \mathrm{H}-3^{\prime}\right), 1.99(\mathrm{dq}, \mathrm{J}=7.5,1.5 \mathrm{~Hz}$, $\left.3 \mathrm{H}, \mathrm{H}-4^{\prime}\right), 1.92$ (dq, J = 1.5, $\left.1.5 \mathrm{~Hz}, 3 \mathrm{H}, \mathrm{H}-2^{\prime} \mathrm{Me}\right)$. ${ }^{13} \mathrm{C}$ NMR: phenylpropane moiety, $\delta_{\mathrm{C}}=195.0(\mathrm{C}=$ O), 149.0 (C-7a), 143.7 (C-4), 140.1 (C-3a), 129.0 (C-6), 109.3 (C-7), 102.9 (C-5), 102.4 (C-2), 70.9 $(\mathrm{C}-\alpha), 56.6(\mathrm{OMe}), 17.3$ (C- $\beta$ ); angeloyl moiety, $\delta_{\mathrm{C}}=167.3\left(\mathrm{C}-1^{\prime}\right), 139.1\left(\mathrm{C}-3^{\prime}\right), 127.1\left(\mathrm{C}-2^{\prime}\right), 20.4$ (C-2'Me), 15.8 (C-4'); signal assignments were verified by HSQC and HMBC experiments. $[\alpha]_{\mathrm{D}}^{27}$ $+2.3^{\circ}$ ( $c$ 0.200, $\left.\mathrm{MeOH}\right)$.

2 was obtained as a pale yellow oil; $[\alpha]_{D}^{15}-28^{\circ}$ (c 0.200, $\mathrm{CHCl}_{3}$ ); UV (MeOH) $\lambda_{\max }(\log \varepsilon) 214$ (4.23) nm; IR (film) $v_{\max } 3430$ (br), 2966, 2931, 2849, 1696, 1649, 1449, 1437, 1261, 1232, 1155, 1132 $\mathrm{cm}^{-1}$; ${ }^{1} \mathrm{H}$ and ${ }^{13} \mathrm{C}$ NMR in Tables I-II; on-line APCIMS $m / z 419[\mathrm{M}+\mathrm{H}]^{+}(5), 401\left[\mathrm{M}-\mathrm{H}_{2} \mathrm{O}+\mathrm{H}\right]^{+}$ (100), $301\left[\mathrm{M}-\text { hemiterpenoyl }-2 \mathrm{H}_{2} \mathrm{O}+\mathrm{H}\right]^{+}$ (16), 261 [M - hemiterpenoyl - isopropanoxy - 
$\left.\mathrm{H}_{2} \mathrm{O}+\mathrm{H}\right]^{+}(90), 161[\mathrm{M}-2$ hemiterpenoyl - isopropanoxy $\left.-2 \mathrm{H}_{2} \mathrm{O}+\mathrm{H}\right]^{+}$(96); HREIMS $m / z$ $441.2620[\mathrm{M}+\mathrm{Na}]^{+}$(calcd. for $\mathrm{C}_{25} \mathrm{H}_{38} \mathrm{O}_{5} \mathrm{Na}$, 441.2617).

3 was obtained as a pale yellow oil; $[\alpha]_{\mathrm{D}}^{27}-57^{\circ}$ (c $\left.0.200, \mathrm{CHCl}_{3}\right)$; UV $(\mathrm{MeOH}) \lambda_{\max }(\log \varepsilon) 214$ (4.45) nm; IR (film) $v_{\max } 3510$ (br), 2962, 2925, 2856, 1709, 1650, 1440,1381, 1268, 1135, 1073, 1015, $982 \mathrm{~cm}^{-1} ;{ }^{1} \mathrm{H}$ and ${ }^{13} \mathrm{C}$ NMR in Tables I-II; on-line APCIMS $m / z 419[\mathrm{M}+\mathrm{H}]^{+}(6), 401[\mathrm{M}-$ $\left.\mathrm{H}_{2} \mathrm{O}+\mathrm{H}\right]^{+}(64), 319\left[\mathrm{M}-\right.$ hemiterpenoyl $-\mathrm{H}_{2} \mathrm{O}$ $+\mathrm{H}]^{+}(44), 301\left[\mathrm{M}-\right.$ hemiterpenoyl $-2 \mathrm{H}_{2} \mathrm{O}+$ $\mathrm{H}]^{+}$(16), 261 [M - hemiterpenoyl - isopropanoxy $\left.-\mathrm{H}_{2} \mathrm{O}+\mathrm{H}\right]^{+}(100), 161[\mathrm{M}-2$ hemiterpenoyl - isopropanoxy $\left.-2 \mathrm{H}_{2} \mathrm{O}+\mathrm{H}\right]^{+}(90)$; HREIMS $m / z 441.2633[\mathrm{M}+\mathrm{Na}]^{+}$(calcd. for $\mathrm{C}_{25} \mathrm{H}_{38} \mathrm{O}_{5} \mathrm{Na}$, 441.2617).

4 was obtained as a pale yellow oil; $[\alpha]_{\mathrm{D}}^{27}$ strongly positive [between +40 and $+60^{\circ}\left(c 0.029, \mathrm{CHCl}_{3}\right)$ ], no exact result is given because of the low concentration; UV (MeOH) $\lambda_{\max }(\log \varepsilon) 208(4.24) \mathrm{nm}$; IR (film) $v_{\max } 3420$ (br), 2968, 2921, 2848, 1704, 1690, 1645, 1443, 1383, 1261, $1139 \mathrm{~cm}^{-1} ;{ }^{1} \mathrm{H}$ and

Allinger N. L., Yuh Y. H. and Lii J.-H. (1989), Molecular mechanics. The MM3 force field for hydrocarbons. J. Amer. Chem. Soc. 111, 8551-8566.

Barrero A. F., Herrador M. M., Quilez J. F., AlvarezManzaneda R., Portal D., Gavin J. A., Gravalos D. G., Simmonds M. S. J. and Blaney W. M. (1999), Bioactive sesquiterpenes from Santolina rosmarinifolia subsp. canescens. A conformational analysis of the germacrane ring. Phytochemistry 51, 529-541.

Dawson J. W. (1961), A revision of the genus Anisotome. Univ. Calif. Publ. Bot. 33, 1-98.

Kozawa M., Morita N., and Hata K. (1978), Chemical components of the roots of Anthriscus sylvestris Hoffm. I. Structures of an acyloxycarboxylic acid and a new phenylpropanoid ester, anthriscusin. Yakugaku Zasshi 98, 1486-1490 (in Japanese).

Micovic I. V. and Rajkovic M. (1985), Total synthesis of $( \pm)$ - $\alpha$-angeloyloxylatifolone and its synthetic isomer ( \pm )- $\alpha$-tiglioyloxylatifolone. J. Serb. Chem. Soc. 50, 67-73.

Parsons M. J., Douglass P. and Macmillan B. H. (1998), Current Names for Wild Plants in New Zealand. Manaaki Whenua - Landcare Research, Lincoln.
${ }^{13} \mathrm{C}$ NMR in Tables I-II; HREIMS $\mathrm{m} / \mathrm{z} 457.2588$ $[\mathrm{M}+\mathrm{Na}]^{+}$(calcd. for $\mathrm{C}_{25} \mathrm{H}_{38} \mathrm{O}_{6} \mathrm{Na}, 457.2566$ ).

Conformational searching and molecular modeling used PCMODEL V 7.0 (Serena Software, Bloomington Ind., USA). The 'Bonds' method was used to randomly rotate bonds $\mathrm{C}-1-\mathrm{O}, \mathrm{C}-6-$ $\mathrm{O}, \mathrm{C}-7-\mathrm{C}-11, \mathrm{C}-8-\mathrm{O}, \mathrm{C}-11-\mathrm{O}, \mathrm{C}-5-\mathrm{C}-6, \mathrm{C}-6-\mathrm{C}-$ 7, C-7-C-8, C-8-C-9, C-9-C-10 and C-10-C-1 to generate starting conformations. These were minimized with the MM3 force-field (Allinger et al., 1989). Results are given in Table III.

\section{Acknowledgements}

The authors wish to thank E. Burgess for HPLC assistance, F. Hoffmann for helping collect the plant material, I. Juranic for help in obtaining the Serbian literature, S. Sturm for LC-MS measurements, M. Thomas for NMR support and B. Clark for MS measurements. A research grant from the Deutsche Forschungsgemeinschaft (DFG) to $\mathrm{CZ}$ is also gratefully acknowledged, as well as support from the New Zealand Foundation for Research, Science and Technology to NBP.

Perry N. B., Burgess E. J., Lorimer S. D. and van Klink J. W. (1996), Fatty acid anilides as internal standards for high performance liquid chromatographic analyses of Valeriana officinalis L. and other medicinal plants. Phytochem. Anal. 7, 263-268.

Stefanovic M., Mladenovic S., Dermanovic M. and Jeremic D. (1977), Sesquiterpene lactones from Laserpitium siler L. (Umbelliferae). Glasnik Hemijskog Drustva Beograd 42, 639-647.

van Klink J. W., Barlow A. J., Perry N. B. and Weavers R. T. (1999), A new irregular diterpene skeleton from Anisotome flexuosa. Tetrahedron Lett. 40, 1409-1412.

Webb C. J. (1986), Breeding Systems and Relationships in Gingidia and Related Australasian Apiaceae. In: Barlow, B. A. (Ed.), Flora and Fauna of Alpine Australasia. Ages and Origins. CSIRO, Melbourne pp. $382-399$.

Zidorn C., Perry N. B. (submitted), A chemosystematically significant 6,8,11-trihydroxygermacrane derivative from the New Zealand Apiaceae Anisotome pilifera. Biochem. Syst. Ecol.

Zidorn C., Sturm S., van Klink J. W., Dawson J. W. Stuppner H. and Perry N. B. (submitted), Chemosystematic investigations of irregular diterpenes in Anisotome and related New Zealand Apiaceae. Phytochemistry. 